Pacific Journal of Mathematics

METRICATION OF SPACES WITH COUNTABLE LARGE BASIS Gary Fred GruenhaGe And Phillip Lee Zeno 


\title{
METRIZATION OF SPACES WITH COUNTABLE LARGE BASIS DIMENSION
}

\author{
Gary Gruenhage AND PhILlip Zenor
}

\begin{abstract}
With the following results, we generalize known metrization theorems for spaces with large basis dimension 0 i.e., non-archimedian spaces) to the higher dimensions: Theorem. If $X$ is a normal $\Sigma$-space with countable large basis dimension, then $X$ is metrizable. Theorem. If $X$ is a normal $w \Delta$-space with countable large basis dimension, then $X$ is metrizable.
\end{abstract}

I. Introduction. A collection $\Gamma$ of subsets of a set $X$ is said to have rank 1 if whenever $g_{1}$ and $g_{2}$ are in $\Gamma$ with $g_{1} \cap g_{2} \neq \varnothing$ then $g_{1} \subset g_{2}$ or $g_{2} \subset g_{1}$. According to P. J. Nyikos [13], a topological space $X$ has large basis dimension $\leqq n$ (denoted $\operatorname{Bad} X \leqq n$ ) if $X$ has a basis which is the union of $n+1$ rank 1 collections of open sets. $X$ has countable large basis dimension $\left(\operatorname{Bad} X \leqq \aleph_{0}\right)$ if $X$ has a basis which is the union of a countable number of rank 1 collections such that each point of $X$ has a basis belonging to one of the collections (a property which is automatically true in the finite case). $\operatorname{Bad} X$ coincides with Ind $X$ and $\operatorname{dim} X$ for metric spaces.

Spaces having large basis dimension 0 are usually called nonarchimedian spaces. Theorems of Nyikos [11] and A. V. Archangelskii [3] show that a non-archimedian space is metrizable if and only if it is a $\Sigma$-space or a $w \Delta$-space. In this paper we show that these results are valid, under mild assumptions, for the higher dimensions. Our results also improve a result of G. Gruenhage [6], who showed that compact spaces having finite large basis dimension are metrizable.

II. Main results. According to Nyikos [11], a tree of open sets is a collection $\Gamma$ of open sets such that if $g \in \Gamma$, then the set $\left\{g^{\prime} \in\right.$ $\left.\Gamma \mid g^{\prime} \supset g\right\}$ is well-ordered by reverse inclusion; that is, $g \leqq g^{\prime}$ if and only if $g \supset g^{\prime}$. Nyikos shows that the rank 1 collections for spaces with Bad $X \leqq \aleph_{0}$ can be considered as rank 1 trees of open sets. The following fact will be used in our proofs:

Lemma 1. Let $T$ be a rank 1 tree of open subsets of a regular space $X$ which contains a basis at each point of a subset $X^{\prime}$ of $X$. Then if $\mathscr{C}$ is a cover of $X^{\prime}$ by open subsets of $X$, there exists a subset $T^{\prime}$ of $T$ such that

(i) $T^{\prime}$ is a cover of $X^{\prime}$;

(ii) the elements of $T^{\prime}$ are pairwise disjoint; 
(iii) $t \in T^{\prime}$ implies that either $t$ is degenerate or $\bar{t}$ is a proper subset of some member of $\mathscr{W}$.

Proof. Put $t$ in $T^{\prime}$ if and only if (a) either $t$ is degenerate or there is a member $U$ of $\mathscr{U}$ such that $\bar{t}$ is a proper subset of $U$ and (b) there is no predecessor of $t$ in $T$ whose closure is a proper subset of some element of $\mathscr{U}$. Since $T$ contains a basis at each point of $X^{\prime}$ and since the predecessors of a given $t \in T$ are well-ordered, it is easy to see that $T^{\prime}$ covers $X^{\prime}$. Further, since $T$ is a tree, the members of $T^{\prime}$ are mutually exclusive.

Nyikos calls a space basically screenable if it has a basis which is the union of countably many rank 1 trees of open sets. Every space $X$ with $\operatorname{Bad} X \leqq \boldsymbol{\aleph}_{0}$ is basically screenable. Basically screenable spaces are, of course, screenable; that is, every open cover has a $\sigma$-pairwise disjoint open refinement. While the following result is known, for the sake of completeness, we include its easy proof:

LEMma 2. A screenable countably compact space $X$ is compact $|2|$.

Proof. Let $\mathscr{C}$ be an open cover of $X$ and let $\mathscr{V}=U\left\{\mathscr{C}_{n} \mid n=\right.$ $1,2, \cdots\}$ be an open refinement of $U$ covering $X$ such that, for each $i$, the members of $\mathscr{V}_{i}$ are mutually exclusive. The set $\left\{V_{n}=\mathbf{U} \mathscr{V}_{n} \mid n=\right.$ $1,2, \cdots\}$ is a countable open cover of $X$; hence, there exists a finite subcover $\left\{V_{n_{1}}, V_{n_{2}}, \cdots, V_{n_{k}}\right\}$. Then $\mathscr{V}_{n_{1}} \cup \mathscr{V}_{n_{2}} \cup \cdots \cup \mathscr{V}_{n_{k}}$ is a point-finite refinement of $\mathscr{U}$. Thus, $X$ is metacompact and it is well-known that a metacompact countably compact space is compact.

According to C. R. Borges [4], a space $X$ is a $w \Delta$-space if there is a sequence $\mathscr{G}_{1}, \mathscr{G}_{2}, \ldots$ of open covers of $X$ such that whenever $x \in X$ and $x_{n} \in \operatorname{St}\left(x, \mathscr{G}_{n}\right)$ for each $n$, then $\left\{x_{1}, x_{2}, \cdots\right\}$ has a cluster point.

THeOREM 1. If $X$ is a regular ws-space with countable large basis dimension, then $X$ has a point countable basis.

Proof. Let $\mathscr{G}_{1}, \mathscr{G}_{2}, \ldots$ be a sequence of open covers of $X$ satisfying the properties given in the definition of a $w \Delta$-space. Let $\mathscr{B}_{1}$, $\mathscr{B}_{2}, \cdots$ and $X_{1}, X_{2}, \cdots$ be sequences such that $X=\mathrm{U}\left\{X_{i} \mid i=1,2, \cdots\right\}$ and, for each $i, \mathscr{B}_{i}$ is a rank 1 tree of open sets containing a basis at each point of $X_{i}$.

For each $i<\omega_{0}$ and $\alpha<\omega_{1}$, we construct a collection $\mathscr{B}(i, \alpha)$ as follows: let $\mathscr{B}(i, 1)$ be a collection of mutually exclusive members of $\mathscr{B}_{i}$ that refines $\mathscr{G}_{1}$ and covers $X_{i}$.

Suppose $\mathscr{B}(i, \beta)$ has been defined for $\beta<\alpha$. If $\alpha$ is not a limit ordinal, applying Lemma 1 , let $\mathscr{B}(i, \alpha)$ be a collection of mutually 
exclusive members of $\mathscr{B}_{i}$ such that

(i) if $j<\omega_{0}$, then $\mathscr{B}(i, j)$ refines $\mathscr{G}_{j}$;

(ii) $\mathscr{B}(i, \alpha)$ covers $(\cup \mathscr{B}(i, \alpha-1)) \cap X_{i}$;

and (iii) $g \in \mathscr{B}(i, \alpha)$ implies $\bar{g}$ is a proper subset of some member of $\mathscr{B}(i, \alpha-1)$, or $g$ is degenerate. If $\alpha$ is a limit ordinal, for each $x \in X_{i}$, let $B(\alpha, x)=\operatorname{Int}\left(\bigcap_{\beta<\alpha}\{g \in \mathscr{S}(i, \beta) \mid x \in g\}\right)$. Note that if $x$ and $y$ are in $X_{i}$, then either $B(\alpha, x)=B(\alpha, y)$ or $B(\alpha, x) \cap B(\alpha, y)=\varnothing$. Let $\mathscr{P}(i, \alpha)=\left\{B(\alpha, x) \mid x \in X_{\imath}\right\}$.

Let $\mathscr{S}_{i}^{*}=\bigcup_{\alpha<\omega_{1}} \mathscr{B}(i, \alpha)$. We will show that $\mathscr{S}_{i}^{*}$ is a point countable collection forming a basis for $X_{i}$ in $X$.

We will say that $g$ is a chain in . ${ }^{*}$ if $g$ is a function from an initial segment of $\omega_{1}$ into $\mathscr{B}_{i}^{*}$ so that (1) $g(\alpha) \in \mathscr{B}(i, \alpha)$ and (2) if $\beta<\alpha$, then $g(\beta) \supset g(\alpha)$. Note that by our construction, if $\beta<\alpha$, then $g(\beta) \supset \overline{g(\alpha)}$. Furthermore, if $x \in X_{i}$, then there is exactly one maximal chain, say $g$, such that $g(\alpha)$ contains $x$ for every $\alpha$ in the domain of $g$.

Claim 1. The domain of each maximal chain in $\mathscr{O}_{i}^{*}$ is countable (and so, $\mathscr{B}_{i}^{*}$ is point countable in $X$ ).

Proof of Claim 1. Suppose the contrary; i.e., there is a chain, say $g$, of length $\omega_{1}$.

Note that $\overline{g\left(\omega_{0}+1\right)}-\bigcap_{\alpha<\omega_{1}} \overline{g(\alpha)}$ is compact. To prove this, we will only show that $\overline{g\left(\omega_{0}+1\right)}-\bigcap_{\alpha<\omega_{1}} \overline{g(\alpha)}$ is countably compact; that $\overline{g\left(\omega_{0}+1\right)}-\bigcap_{\alpha<\omega_{1}} \overline{g(\alpha)}$ is compact will then follow from Lemma 2 . To this end, let $N$ denote a countable subset of $\overline{g\left(\omega_{0}+1\right)}-\bigcap_{\alpha<\omega_{1}} \overline{g(\alpha)}$. There is an $\alpha$ so that $g(\alpha)$ does not meet $N$. In particular then, no point of $\overline{g(\alpha+1)}$ is a limit point of $N$. Because of property (i), it must be the case that $N$ has a limit point in $\overline{g\left(\omega_{0}+1\right)}-\bigcap_{\alpha<\omega_{1}} \overline{g(\alpha)}$; and so, $\overline{g\left(\omega_{0}+1\right)}-\bigcap_{\alpha<\omega_{1}} \overline{g(\alpha)}$ is compact. But, $\left\{\overline{g\left(\omega_{0}+1\right)}-\overline{g(\alpha) \mid} \alpha<\omega_{1}\right\}$ is an open cover of $\overline{g\left(\omega_{0}+1\right)}-\bigcap_{\alpha<\omega_{1}} \overline{g(\alpha)}$ with no finite subcover, which is a contradiction from which Claim 1 follows.

Claim 2: $\mathscr{D}_{1}^{*}$ is a basis for $X_{2}$ in $X$; in particular, if $x \in X_{i}$ and $g$ is the maximal chain in $\mathscr{B}_{i}^{*}$ centered at $x$, then $\{g(\alpha) \mid \alpha$ is in the domain of $g\}$ is a local basis for $x$ in $X$.

Proof of Claim 2. Suppose otherwise. Then there is a point $x$ of $X_{i}$ so that the maximal chain, $g$, centered at $x$ does not yield a basis at $x$ in $X$; i.e., $\{g(\alpha) \mid \alpha \in$ domain of $g\}$ is not a local basis for $x$ in $X$. Since the domain of $g$ is countable, there is a first $\alpha_{0}<\omega_{1}$ not in the domain $g$. There is a member $B$ of $\mathscr{B}_{i}$ so that if $\alpha<\alpha_{0}$, then $g(\alpha)$ is not a subset of $B$ but this means that $B$ is a subset of each $g(\alpha)$. Then $x$ is in the interior of $\bigcap_{\alpha<\alpha_{0}} g(\alpha)$. Thus, by our 
construction of $\mathscr{B}\left(i, \alpha_{0}\right)$, there is a member of $\mathscr{B}\left(i, \alpha_{0}\right)$ that contains $x$. This contradicts the maximality of $g$ and it follows that $\{g(\alpha) \mid \alpha$ is in the domain of $g$ \} is a local basis for $x$ in $X$.

We now have that $\bigcup_{i<\omega_{0}} \mathscr{B}_{i}^{*}$ is a point countable basis for $X$.

If $\mathscr{H}$ is a cover of the space $X$ and if $x \in X$, then $C(x, H)$ will denote the set $\cap\{H \in \mathscr{H} \mid x \in H\}$. According to K. Nagami [9], the space $X$ is a $\Sigma$-space if there is a sequence $\mathscr{F}_{1}, \mathscr{F}_{2}, \ldots$ of locally finite closed covers of such that if $x_{0}, x_{1}, x_{2}, \ldots$ is a sequence with $x_{i} \in C\left(x_{0}, \mathscr{F}_{i}\right)$ for each $0<i<\omega_{0}$, then $\left\{x_{i}\right\}$ has a cluster point. The sequence $\mathscr{F}_{1}, \mathscr{F}_{2}, \cdots$ is called a spectral $\Sigma$-sequence for $X$.

We will, without loss of generality, assume that each $\mathscr{F}_{i}$ is closed under intersections and, for each $i, \mathscr{F}_{i+1}$ refines $\mathscr{F}_{i}$.

Lemma 3. If $X$ is a space with countable large basis dimension such that each uncountable subset of $X$ has a limit point, then $X$ is Lindelof.

Proof. Since $X$ has countable large basis dimension, $X$ is screenable. G. Aquaro [1] has proved that every meta-Lindelof (and thus every screenable) space in which every uncountable set has a limit point is Lindelof.

THEOREM 2. If $X$ is a regular $\Sigma$-space with countable large basis dimension then $X$ has a point countable basis.

Proof. Let $\mathscr{F}_{1}, \mathscr{F}_{2}, \ldots$ be a sequence of locally finite closed coverings of $X$ given in the definition of a $\Sigma$-space. For each $n$, let $\mathscr{G}_{n}$ be an open cover of $X$ such that each member of $\mathscr{G}_{n}$ intersects only finitely many members of $\mathscr{F}_{n}$. Let $\mathscr{B}_{1}, \mathscr{B}_{2}, \cdots$ and $X_{1}, X_{2}, \ldots$ be sequences such that $X=\bigcup_{i<\omega_{0}} X_{i}$ and $\mathscr{B} i$ is a rank 1 tree of open sets which contains a basis for each point of $X_{i}$.

Define $\mathscr{B}(i, \alpha), i<\omega_{0}, \alpha<\omega_{1}$, exactly as in the proof of Theorem 1. Let $\mathscr{B}_{i}^{*}=\bigcup_{\alpha<\omega_{1}} \mathscr{B}(i, \alpha)$ and define chain in $\mathscr{B}_{i}^{*}$ as in the proof to Theorem 1.

Claim 1. Every chain in $\mathscr{B}_{i}^{*}$ is countable.

Proof of Claim 1. Suppose otherwise; i.e., suppose that $g$ is a chain in $\mathscr{B}_{i}^{*}$ with length $\omega_{1}$. Let $K=\bigcap_{\alpha<\omega_{1}} \overline{g(\alpha)}$. Every uncountable of $\overline{g\left(\omega_{0}\right)}-K$ has a limit point in $\overline{g\left(\omega_{0}\right)}-K$ for suppose otherwise; that is, suppose that $H$ is an uncountable subset of $\overline{g\left(\omega_{0}\right)}-K$ with no limit point in $\overline{g\left(\omega_{0}\right)}-K$.

Suppose that there is a point, $h$, of $H$ such that, for each $n$, 
$C\left(h, \mathscr{F}_{n}\right)$ intersects infinitely many points of $H$. Then there is a countable subset $N$ of $H$ with a limit point. Since $N$ is countable, there is an $\alpha<\omega_{1}$ so that $g(\alpha)$ does not intersect $N$. It follows that no point of $K$ is a limit point of $N$. Hence, no point of $K$ is a limit point of $N$; and so, $H$ has a limit point in $\overline{g\left(\omega_{0}\right)}-K$. This is a contradiction from which it follows that, for each $h$ in $H$, there is an integer $n(h)$ such that $C(h, n(h))$ intersects only finitely many members of $H$. Thus, there is an $N$ and an uncountable subset $H^{*}$ of $H$ so that if $h \in H^{*}$, then $n(h)=N$ and $\left\{C\left(h, F_{N}\right) \mid h \in\right.$ $\left.H^{*}\right\}$ is an infinite subcollection of $\mathscr{F}_{N}$, each member of which intersects $g(N)$. But, $g(N)$ is in $\mathscr{B}(i, N)$ which contradicts the fact that $\mathscr{B}(i, N)$ refines $\mathscr{G}_{N}$. It follows that each uncountable subset of $\overline{g\left(\omega_{0}\right)}-K$ has a limit point in $\overline{g\left(\omega_{0}\right)}-K$; and so, by Lemma $3, \overline{g\left(\omega_{0}\right)}-$ $K$ is Lindelof. But $\left\{\overline{g\left(\omega_{0}\right)}-\overline{g(\alpha)} \mid \alpha<\omega_{1}\right\}$ is an open cover of $\overline{g\left(\omega_{0}\right)}-$ $K$ with no countable subcover which is a contradiction from which Claim 1 follows.

That $\mathscr{B}_{i}^{*}$ contains a basis at each point of $X_{i}$ follows exactly as in the proof of Theorem 1. Thus Theorem 2 is proved.

THEOREM 3. If $X$ is a normal $\Sigma$-space with countable large basis dimension, then $X$ is metrizable.

Proof. R. E. Hodel has proved that every $\Sigma$-space is a $\beta$-space [8], and that every $\beta$-space is countably metacompact [7]. A screenable countably metacompact space is metacompact. Nagami [10] has shown that a normal screenable metacompact space is paracompact. But a paracompact $\Sigma$-space with a point-countable base is metrizable [9].

THEOREM 4. If $X$ is a normal wA-space with countable large basis dimension, then $X$ is metrizable.

Proof. As above, $X$ is normal, screenable, and metacompact (since every $w \Delta$-space is a $\beta$-space), hence paracompact. But a papacompact $w \Delta$-space is an $M$-space, hence a $\Sigma$-space. Thus $X$ is metrizable.

\section{REFERENCES}

1. G. Aquaro, Point-countable open coverings in countably compact spaces, General, Topology and Its Relations to Modern Analysis and Algebra II, Academia, Prague (1966), 39-41.

2. R. Arens and J. Dugundji, Remark on the concept of compactness, Portugaliae Math., 9 (1950), 141-143.

3. A. V. Arhangel'skii and V. V. Filippov, Spaces with bases of finite rank, Math. USSR Sb., 16 (1972), 147-158. 
4. C. J. R. Borges, On metrizability of topological spaces, Canad, J. Math., 20 (1968), 795-804.

5. J. Dugundji, Topology, Allyn and Bacon, 1966.

6. G. Gruenhage, Large basis dimension and metrizability, to appear in Proc. AMS.

7. R. E. Hodel, Spaces defined by sequences of open covers which guarantee that certain sequences have cluster points, Duke Math. J., 39 (1972), 253-263.

8. — Spaces characterized by sequences of covers which guarantee that certain sequences have cluster points, Proceedings of the U. of Houston Point Set Topology Conference (1971), 105-114.

9. K. Nagami, $\Sigma$-spaces, Fund. Math., 65 (1969), 169-192.

10. - Paracompactness and strong screenability, Nagoya Math. J., 8 (1955), 83-88.

11. P. J. Nyikos, Some surprising base properties in topology, Studies in topology, New York, Academic Press, 1975.

12. T. Shiraki, M-spaces, their generalization, and metrization theorems, Sci. Rep. Tokyo Kyoiku Daigaku Ser. A, 11 (1971), 57-67.

Received January 22, 1975 and in revised form June 3, 1975.

AUBURN UNIVERSity 


\title{
PACIFIC JOURNAL OF MATHEMATICS
}

\section{EDITORS}

RichaRd ARENS (Managing Editor)

University of California

Los Angeles, California 90024

\section{R. A. Beaumont}

University of Washington

Seattle, Washington 98105
J. DUGUNDJI

Department of Mathematics University of Southern California Los Angeles, California 90007

D. Gilbarg and J. Milgram

Stanford University

Stanford, California 94305

\section{ASSOCIATE EDITORS}
E. F. BECKENBACH
B. H. NeUmanN
F. WOLF
K. YoshIDA

\section{SUPPORTING INSTITUTIONS}

\author{
UNIVERSITY OF BRITISH COLUMBIA \\ CALIFORNIA INSTITUTE OF TECHNOLOGY \\ UNIVERSITY OF CALIFORNIA \\ MONTANA STATE UNIVERSITY \\ UNIVERSITY OF NEVADA \\ NEW MEXICO STATE UNIVERSITY \\ OREGON STATE UNIVERSITY \\ UNIVERSITY OF OREGON \\ OSAKA UNIVERSITY
}

\author{
UNIVERSITY OF SOUTHERN CALIFORNIA \\ STANFORD UNIVERSITY \\ UNIVERSITY OF TOKYO \\ UNIVERSITY OF UTAH \\ WASHINGTON STATE UNIVERSITY \\ UNIVERSITY OF WASHINGTON \\ AMERICAN MATHEMATICAL SOCIETY
}

The Supporting Institutions listed above contribute to the cost of publication of this Journal, but they are not owners or publishers and have no responsibility for its content or policies.

Mathematical papers intended for publication in the Pacific Journal of Mathematics should be in typed form or offset-reproduced, (not dittoed), double spaced with large margins. Underline Greek letters in red, German in green, and script in blue. The first paragraph or two must be capable of being used separately as a synopsis of the entire paper. Items of the bibliography should not be cited there unless absolutely necessary, in which case they must be identified by author and Journal, rather than by item number. Manuscripts, in triplicate, may be sent to any one of the editors. Please classify according to the scheme of Math. Reviews, Index to Vol. 39. All other communications should be addressed to the managing editor, or Elaine Barth, University of California, Los Angeles, California, 90024.

The Pacific Journal of Mathematics expects the author's institution to pay page charges, and reserves the right to delay publication for nonpayment of charges in case of financial emergency.

100 reprints are provided free for each article, only if page charges have been substantially paid. Additional copies may be obtained at cost in multiples of 50 .

The Pacific Journal of Mathematics is issued monthly as of January 1966. Regular subscription rate: $\$ 72.00$ a year (6 Vols., 12 issues). Special rate: $\$ 36.00$ a year to individual members of supporting institutions.

Subscriptions, orders for back numbers, and changes of address should be sent to Pacific Journal of Mathematics, 103 Highland Boulevard, Berkeley, California, 94708.

PUBLISHED BY PACIFIC JOURNAL OF MATHEMATICS, A NON-PROFIT CORPORATION

Printed at Kokusai Bunken Insatsusha (International Academic Printing Co., Ltd.), 8-8, 3-chome, Takadanobaba, Shinjuku-ku, Tokyo 160, Japan.

Copyright (C) 1975 by Pacific Journal of Mathematics Manufactured and first issued in Japan 


\section{Pacific Journal of Mathematics}

\section{Vol. 59, No. $2 \quad$ June, 1975}

Aharon Atzmon, A moment problem for positive measures on the unit disc ........

Peter W. Bates and Grant Bernard Gustafson, Green's function inequalities for

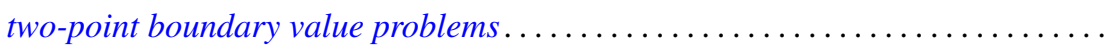

Howard Edwin Bell, Infinite subrings of infinite rings and near-rings ...........

Grahame Bennett, Victor Wayne Goodman and Charles Michael Newman, Norms of

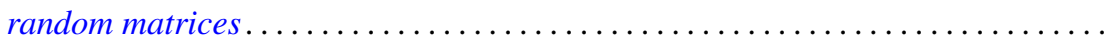

Beverly L. Brechner, Almost periodic homeomorphisms of $E^{2}$ are periodic.........

Beverly L. Brechner and R. Daniel Mauldin, Homeomorphisms of the plane ........

Jia-Arng Chao, Lusin area functions on local fields ......................

Frank Rimi DeMeyer, The Brauer group of polynomial rings ...............

M. V. Deshpande, Collectively compact sets and the ergodic theory of

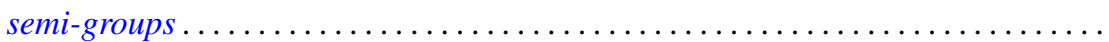

Raymond Frank Dickman and Jack Ray Porter, $\theta$-closed subsets of Hausdorff

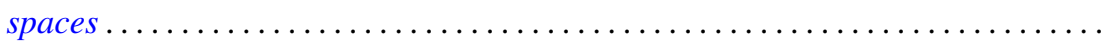

Charles P. Downey, Classification of singular integrals over a local field .......... 407

Daniel Reuven Farkas, Miscellany on Bieberbach group algebras . . . . . . . . . . . .

Peter A. Fowler, Infimum and domination principles in vector lattices . . . . . . . . . .

Barry J. Gardner, Some aspects of T-nilpotence. II: Lifting properties over

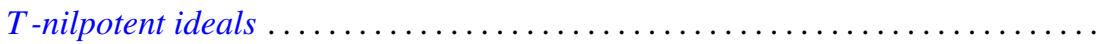

Gary Fred Gruenhage and Phillip Lee Zenor, Metrization of spaces with countable

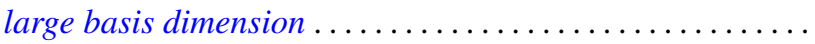

J. L. Hickman, Reducing series of ordinals...

Hugh M. Hilden, Generators for two groups related to the braid group ...

Tom (Roy Thomas Jr.) Jacob, Some matrix transformations on analytic sequence

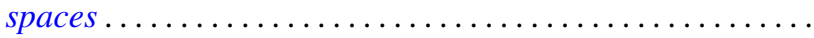

Elyahu Katz, Free products in the category of $k_{w}$-groups . .....

Tsang Hai Kuo, On conjugate Banach spaces with the Radon-Nikodým property...

Norman Eugene Liden, $K$-spaces, their antispaces and related mappings ...

Clinton M. Petty, Radon partitions in real linear spaces ........

Alan Saleski, A conditional entropy for the space of pseudo-Menger maps ....

Michael Singer, Elementary solutions of differential equations .

Eugene Spiegel and Allan Trojan, On semi-simple group algebras. I. . .

Charles Madison Stanton, Bounded analytic functions on a class of open Riemann

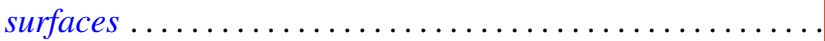

Sherman K. Stein, Transversals of Latin squares and their generalizations ....

Ivan Ernest Stux, Distribution of squarefree integers in non-linear sequences . . .

Lowell G. Sweet, On homogeneous algebras ................

Lowell G. Sweet, On doubly homogeneous algebras .......... .

Florian Vasilescu, The closed range modulus of operators ......

Arthur Anthony Yanushka, A characterization of the symplectic groups $\operatorname{PSp}(2 m, q)$

as rank 3 permutation groups... 